\title{
ON THE ENUMERATION OF PLANAR TREES OF HEXAGONS
}

\author{
by L. W. BEINEKE and R. E. PIPPERT
}

(Received 5 June, 1973)

1. Introduction. In their paper "The enumeration of tree-like polyhexes", Harary and Read [6] consider structures obtained by assembling hexagons subject to certain restrictions. Their problem is introduced as a simplified hexagonal cell-growth problem.

In effect, the following inductive definition of polyhexes is equivalent to theirs. A single hexagon is a polyhex, and a polyhex with $n+1$ hexagons is obtained from one with $n$ hexagons by adjoining a new hexagon along an edge so that no vertex is on more than two hexagons. It is understood that the new hexagon has only the one edge in common with the given polyhex and that two hexagons that meet share an entire edge.

The polyhexes with up to four hexagons are shown in Figure 1(a). We observe that the definition precludes three hexagons having a vertex in common as well as " rings" of hexagons. Harary and Read stipulate that the hexagons be regular, but this requirement is not used in the enumeration.

As observed by Harary and Read, these structures may be regarded in various ways: as hexagonal animals in a cell-growth problem, as hexagonal variations of polyominoes, and as certain chemical compounds. Another possible interpretation is that they are dissections of polygons into hexagons by means of nonintersecting diagonals, no two having a common vertex. The number of vertices in a polygon that can be dissected into hexagons must be congruent to 2 modulo 4 . Figure 2(a) shows the two dissections of a 14-gon subject to these restrictions. These correspond to the two polyhexes with three hexagons; of course, regularity and convexity of the polygon are not important, although one cannot in general use an arbitrary drawing of a polygon.

It is in this context of polygon dissections that we were led to other enumeration problems involving structures composed of hexagons. It has been observed $[1,5]$ that planar 2-trees (composed of triangles) are equivalent to arbitrary dissections of polygons into triangles. This suggests consideration of other dissections as "trees of $n$-gons". In particular, we shall consider "trees of hexagons", which are more general than polyhexes.

We define planar hexagon trees inductively. A single edge and a single hexagon are planar hexagon trees, and a planar hexagon tree with $n+1$ hexagons is obtained from one with $n$ hexagons by adding a new hexagon along an edge on only one hexagon. In this paper we shall simply call these hexagon trees. A more general class of structures, which are also tree-like but which do not have this property of planarity, have been enumerated by Palmer [8] in the labelled case.

Figure 1 shows all hexagon trees with up to four hexagons. We observe that a hexagon tree can be drawn in the plane with, possibly, irregular hexagons or overlapping hexagons. The definition precludes any cycles of hexagons. As we indicated above, hexagon trees are equivalent to dissections of $(4 n+2)$-gons into $n$ hexagons by means of nonintersecting diagonals 


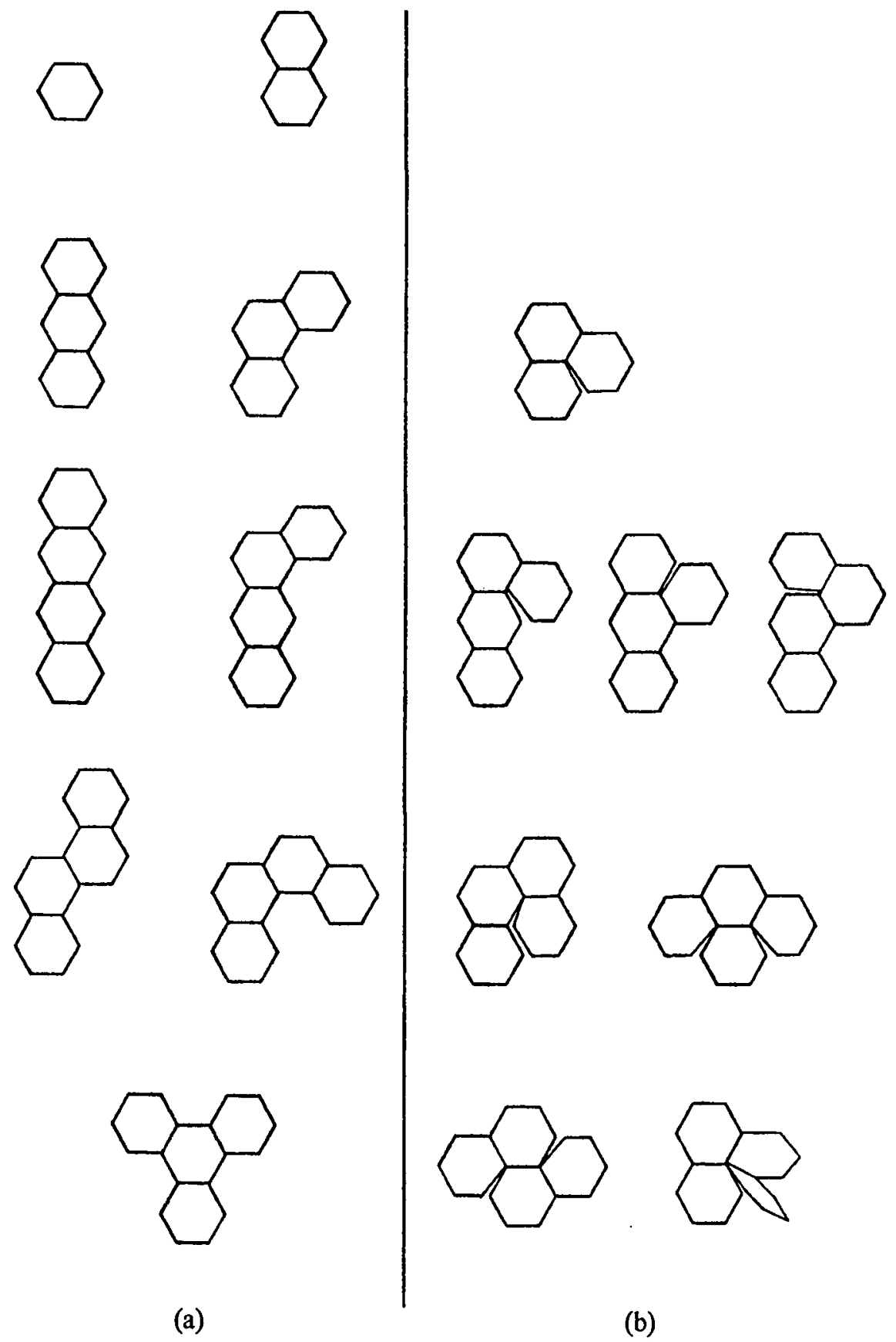

FIGURE 1. 


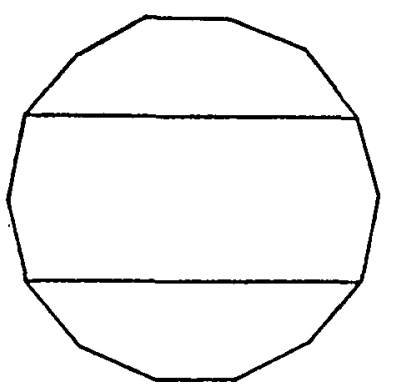

(a)
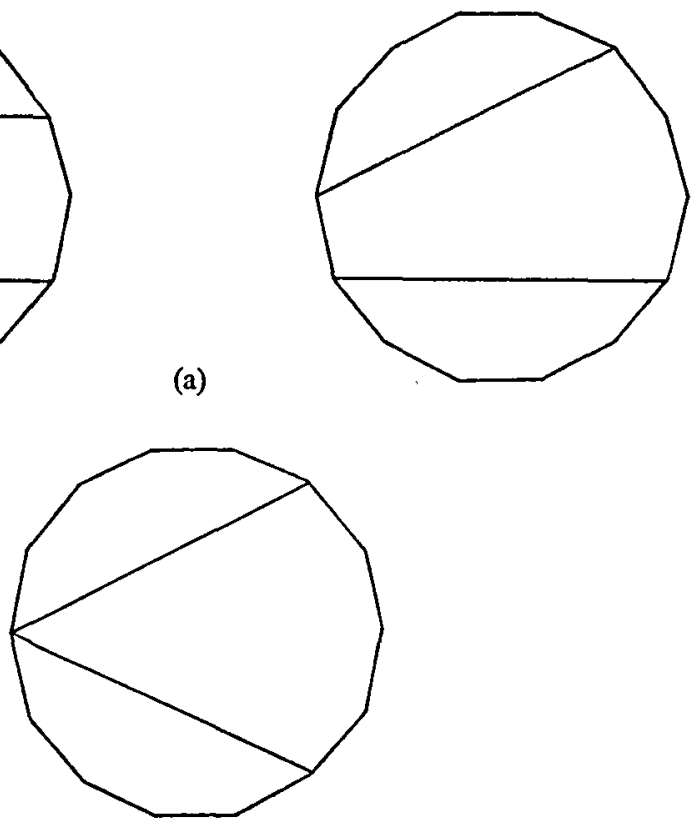

(b)

FIGURE 2.

(more than one diagonal at a vertex now being allowed). Figure 2 shows the three dissections of a 14-gon corresponding to hexagon trees.

Clearly hexagon trees include polyhexes as a subclass, there being no restriction on the number of hexagons that can meet at a vertex. Some other interesting structures form a subclass of polyhexes (and hence of hexagon trees); we define a hexagon chain to be a polyhex in which no hexagon meets more than two other hexagons. All polyhexes in Figure 1(a) are hexagon chains except the last.

The primary objective of this paper is to obtain expressions in closed form for the numbers of hexagon trees and of hexagon chains. This is in contrast to the result for polyhexes, in which the number is obtained only in generating function form.

We obtain the number of hexagon trees by counting those with each of the possible automorphism groups. This method has been used by Guy [4] in enumerating triangulations of a polygon and also by the authors [3] in enumerating dissections of polyhedra into tetrahedra. A survey of both of these results is found in [2]. In each case the enumeration of unrooted structures utilizes the number having a particular kind of rooting.

In $\$ 2$ we determine all possible automorphism groups of hexagon trees. In $\$ 3$, we obtain formulas first for rooted hexagon trees, then for hexagon trees with each possible group, concluding with an expression in closed form for the number of unrooted hexagon trees. $\$ 4$ provides similar results, but with less detail, for hexagon chains. We conclude the paper with a discussion in $\$ 5$ of other aspects of the enumeration of polyhexes. 
2. Automorphism groups. Before determining the automorphism groups, we first determine all possible automorphisms of hexagon trees. To facilitate the description of the various types of symmetry, we introduce some notation. Lower-case Greek letters will denote edges of a hexagon, except that $\imath$ will denote the identity permutation. In the case of symmetry involving the interchange of a pair of hexagons with a common edge, edges in one will be designated with primes. These conventions are indicated in Figure 3.
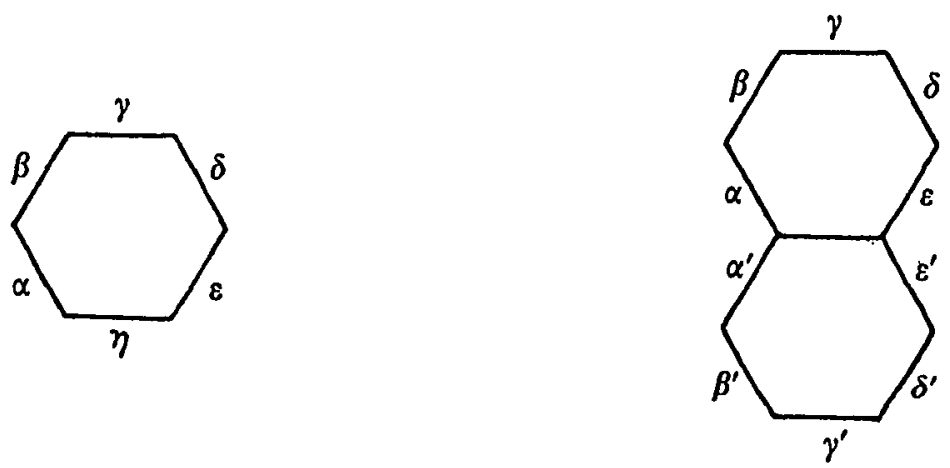

FIGURE 3.

Table 1 lists the possible types of automorphisms; in each of the first six, there is a fixed hexagon, while in each of the last two, two adjacent hexagons are interchanged. Each is simply a rotation or a reflection.

TABLE 1. AUTOMORPHISM TYPES

\begin{tabular}{ll}
$\quad$ Name & \multicolumn{1}{c}{ Example } \\
1. Identity & $(\alpha \varepsilon)(\beta \delta)(\gamma)(\eta)$ \\
2. Side reflection & $(\alpha \beta)(\gamma \eta)(\delta \varepsilon)$ \\
3. Angle reflection & $(\alpha \delta)(\beta \varepsilon)(\gamma \eta)$ \\
4. Digonal rotation & $(\alpha \gamma \varepsilon)(\beta \delta \eta)$ \\
5. Trigonal rotation & $(\alpha \beta \gamma \delta \varepsilon \eta)$ \\
6. Hexagonal rotation & $\left(\alpha \alpha^{\prime}\right)\left(\beta \beta^{\prime}\right)\left(\gamma \gamma^{\prime}\right)\left(\delta \delta^{\prime}\right)\left(\varepsilon \varepsilon^{\prime}\right)$ \\
7. Reversal & $\left(\alpha \varepsilon^{\prime}\right)\left(\beta \delta^{\prime}\right)\left(\gamma \gamma^{\prime}\right)\left(\delta \beta^{\prime}\right)\left(\varepsilon \alpha^{\prime}\right)$ \\
8. Half-turn &
\end{tabular}

In Table 2 we list the thirteen possible groups determined by these automorphisms. We consider first those groups in which every automorphism has a fixed hexagon. There are of course six generated by a single automorphism; it is a routine matter to verify that combinations of automorphisms yield only four others (F, H, I and J below). If two adjacent hexagons are interchanged, there are the two $(\mathrm{K}$ and $\mathrm{L})$ generated by one symmetry and the one (M) generated by two. We note that, of the permutations having a fixed hexagon, only the identity and a side reflection can be in a group in which some automorphisms interchange two adjacent hexagons and some do not (because of the fixed edge).

The diagrams in Figure 4 indicate the type of structure required by each group. In $\mathrm{C}$ to $\mathrm{J}$, the pictured hexagon represents the fixed hexagon to which rooted hexagon trees are attached, 


\section{ENUMERATION OF PLANAR TREES OF HEXAGONS}

TABLE 2. AUTOMORPHISM GROUPS

Generated by
A. Identity
B. Side reflection
C. Angle reflection
D. Digonal rotation
E. Trigonal rotation
F. Side reflection and angle reflection
G. Hexagonal rotation
H. Trigonal rotation and side reflection
I. Trigonal rotation and angle reflection
J. Hexagonal rotation and side reflection
K. Reversal
L. Half-turn
M. Reversal and half-turn

\section{Isomorphic to}

Symmetric, degree 2

Symmetric, degree 2

Symmetric, degree 2

Cyclic, degree 3

Klein 4-group

Cyclic, degree 6

Symmetric, degree 3

Symmetric, degree 3

Dihedral, degree 6

Symmetric, degree 2

Symmetric, degree 2

Klein 4-group
Elements are

$(\alpha \varepsilon)(\beta \delta)(\gamma)(\eta),\llcorner$

$(\alpha \beta)(\gamma \eta)(\delta \varepsilon)$,

$(\alpha \delta)(\beta \varepsilon)(\gamma \eta), \iota$

$(\alpha \gamma \varepsilon)(\beta \delta \eta),(\alpha \varepsilon \gamma)(\beta \eta \delta)$,

$(\alpha \varepsilon)(\beta \delta)(\gamma)(\eta),(\alpha \beta)(\gamma \eta)(\delta \varepsilon)$,

$(\alpha \delta)(\beta \varepsilon)(\gamma \eta)$, ८

$(\alpha \beta \gamma \delta \varepsilon \eta),(\alpha \gamma \varepsilon)(\beta \delta \eta),(\alpha \delta)(\beta \varepsilon)(\gamma \eta)$,

$(\alpha \varepsilon \gamma)(\beta \eta \delta),(\alpha \eta \varepsilon \delta \gamma \beta), \iota$

$(\alpha \gamma \varepsilon)(\beta \delta \eta),(\alpha \varepsilon \gamma)(\beta \eta \delta),(\alpha \varepsilon)(\beta \delta)(\gamma)(\eta)$,

$(\alpha \gamma)(\delta \eta)(\beta)(\varepsilon),(\beta \eta)(\gamma \varepsilon)(\alpha)(\delta), \mathrm{l}$

$(\alpha \gamma \varepsilon)(\beta \delta \eta),(\alpha \varepsilon \gamma)(\beta \eta \delta),(\alpha \beta)(\gamma \eta)(\delta \varepsilon)$,

$(\alpha \delta)(\beta \gamma)(\varepsilon \eta),(\alpha \eta)(\beta \varepsilon)(\gamma \delta)$, ।

All symmetries of a hexagon

$\left(\alpha \alpha^{\prime}\right)\left(\beta \beta^{\prime}\right)\left(\gamma \gamma^{\prime}\right)\left(\delta \delta^{\prime}\right)\left(\varepsilon \varepsilon^{\prime}\right)$, ‘

$\left(\alpha \varepsilon^{\prime}\right)\left(\beta \delta^{\prime}\right)\left(\gamma \gamma^{\prime}\right)\left(\delta \beta^{\prime}\right)\left(\varepsilon \alpha^{\prime}\right)$, ,

$\left(\alpha \alpha^{\prime}\right)\left(\beta \beta^{\prime}\right)\left(\gamma \gamma^{\prime}\right)\left(\delta \delta^{\prime}\right)\left(\varepsilon \varepsilon^{\prime}\right)$,

$(\alpha \varepsilon)\left(\beta \delta^{\prime}\right)(\gamma \gamma)(\delta \beta)\left(\varepsilon \alpha^{\prime}\right)$,

$(\alpha \varepsilon)(\beta \delta)(\gamma)\left(\alpha^{\prime} \varepsilon^{\prime}\right)\left(\beta^{\prime} \delta^{\prime}\right)\left(\gamma^{\prime}\right)$,

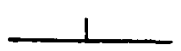

B

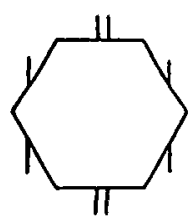

F

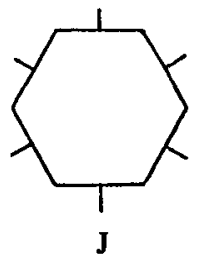

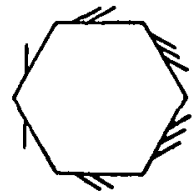

C

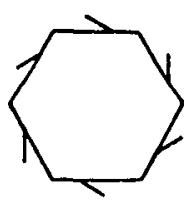

G

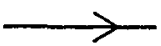

K.

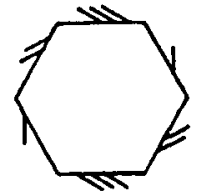

D

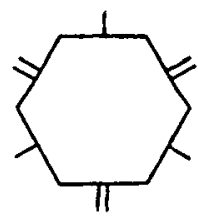

H

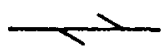

L

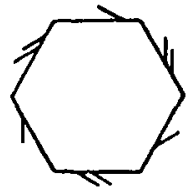

E

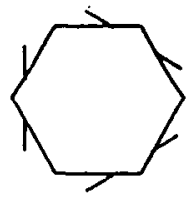

I

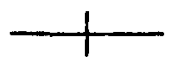

M

Figure 4. 
while in $\mathrm{B}, \mathrm{K}, \mathrm{L}$ and $\mathrm{M}$, the pictured edge has one or two rooted hexagon trees attached. The type of hexagon tree attached at an edge is indicated by the marks on the edge; perpendicular marks indicate trees with reflectional symmetry, slanted half-arrows indicate arbitrary trees. Within a figure, different numbers of marks generally indicate different trees, and the relative directions of slanted marks indicate the relative positions of the trees.

3. Hexagon trees. In this part we enumerate both rooted and unrooted hexagon trees, the former being needed for the latter.

(a) Rooted trees. Rooting a hexagon tree consists of embedding it in the plane with a distinguished exterior edge. (In an embedding, the interiors of distinct hexagons must be disjoint. An edge is exterior if it is on at most one hexagon.) This is equivalent to selecting an exterior edge and assigning an orientation to it. It follows that the number of possible rootings is twice the number of exterior edges divided by the order of the automorphism group. Thus there are five different rooted hexagon trees (see Figure 5) with two hexagons; of course they all come from the same unrooted one. We observe that a rooted hexagon tree is equivalent to a dissection of a fixed polygon into hexagons. The five rooted trees in Figure 5 correspond to the five possible choices of a diagonal dividing a fixed decagon into two hexagons.
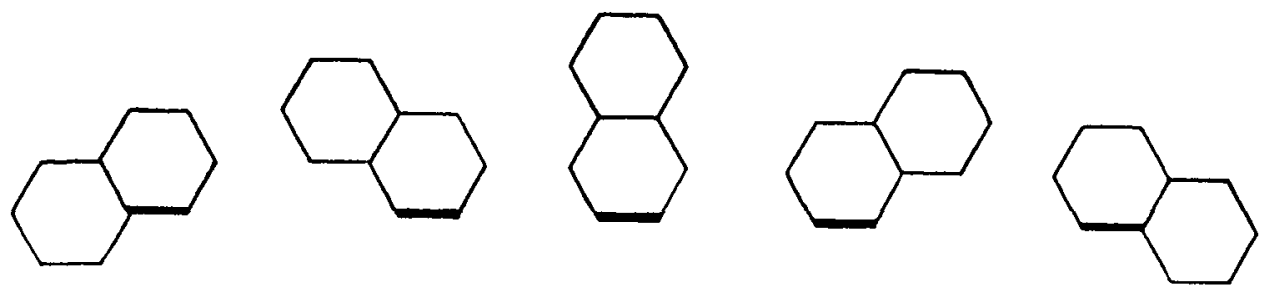

Figure 5.

We let $T(n)$ denote the number of rooted hexagon trees with $n$ hexagons. This number was found by Motzkin [7] as the number of hexagonal dissections of polygons. The same number was independently found by the authors [1] in the enumeration of rooted $k$-trees embeddable in $k$-space, it being the number for $k=5$. In addition to $T(n)$, we require $U(n)$, the number of rooted hexagon trees that are symmetric in the root edge; that is, that remain the same when the orientation of the root edge is reversed. For completeness, we outline a derivation of $T(n)$ and also determine the value of $U(n)$. Some preliminary lemmas will simplify the proofs.

LEMma 1. The numbers $T(k)$ satisfy the relation

$$
T(n)=\sum_{\langle n-1\rangle} \prod_{i=1}^{5} T\left(n_{i}\right),
$$

where the sum is over all partitions of $n-1$ into five non-negative parts $n_{1}, n_{2}, \ldots, n_{5}$. 
Proof. A nontrivial rooted hexagon tree is obtained when one begins with a hexagon having a root edge and then adds a rooted hexagon tree along each of the other five edges. The summation takes into account all possible choices and combinations of rooted trees.

LEMma 2. The numbers $U(k)$ and $T(k)$ satisfy

$$
U(n)=\sum_{2 i+2 j+h=n-1} T(i) T(j) U(h) .
$$

Proof. The following procedure gives a construction for hexagon trees that are symmetric in the root edge. Begin with five rooted trees, of which two pairs are mirror images and the other tree is root-symmetric. Join them to a hexagon having a root edge so that the rootsymmetric one is joined opposite the root edge and the pairs are joined so that the result has root symmetry. Since every hexagon tree with root symmetry can be obtained in this way, one gets the theorem by considering all possible combinations.

The third lemma is a special case of a combinatorial identity due originally to Hagen (see Riordan [9, p. 169]).

Lemma 3. If $A_{k}(\alpha, \beta) \equiv \frac{\alpha}{\alpha+\beta k}\left(\begin{array}{c}\alpha+\beta k \\ k\end{array}\right)$, then

$$
\sum_{i+j=n} A_{i}\left(\alpha_{1}, \beta\right) A_{j}\left(\alpha_{2}, \beta\right)=A_{n}\left(\alpha_{1}+\alpha_{2}, \beta\right) .
$$

We are now ready to obtain formulas for $T(n)$ and $U(n)$.

THEOREM 1. The number $T(n)$ of rooted planar hexagon trees with $n$ hexagons is

$$
T(n)=\frac{1}{5 n+1}\left(\begin{array}{c}
5 n+1 \\
n
\end{array}\right)=A_{n}(1,5) .
$$

The number $U(n)$ having root-symmetry is

$$
U(n)=\left\{\begin{array}{l}
\frac{1}{5 m+1}\left(\begin{array}{c}
5 m+1 \\
m
\end{array}\right)=A_{m}(1,5) \quad \text { if } \quad n=2 m \\
\frac{3}{5 m+3}\left(\begin{array}{c}
5 m+3 \\
m
\end{array}\right)=A_{m}(3,5) \quad \text { if } \quad n=2 m+1 .
\end{array}\right.
$$

Proof. To prove the first statement, we define a generating function

Using Lemma 1, we find that

$$
t(x) \equiv \sum_{n=0}^{\infty} T(n) x^{4 n+1} .
$$

By Lagrange inversion,

$$
t^{5}(x)=t(x)-x
$$

$$
t(x)=\sum_{n=0}^{\infty} \frac{(5 n) !}{n !(4 n+1) !} x^{4 n+1}
$$

from which the result follows. 
In proving the second statement, we consider separately the two cases of even and odd values of $n$.

Case (i): $n=2 m$. We use induction to show that $U(2 m)=T(m)$. Clearly $U(2)=T(1)$. Assume that $U(2 h)=T(h)$ for $h<m$. Two applications of Lemma 2 yield

$$
U(2 m)=\sum_{2 i+2 j+2 k+2 l+2 h=n-2} T(i) T(j) T(k) T(l) U(2 h),
$$

which, by the inductive hypothesis and Lemma 1 , gives $U(2 m)=T(m)$. The result follows from Theorem 1.

Case (ii): $n=2 m+1$. By Lemma 2 and case (i) of this proof,

$$
U(2 m+1)=\sum_{i+j+k=m} T(i) T(j) T(k)
$$

Repeated application of Lemma 3 yields

$$
\begin{aligned}
U(2 m+1) & =\sum_{i+j+k=m} A_{i}(1,5) A_{j}(1,5) A_{k}(1,5) \\
& =A_{m}(3,5)
\end{aligned}
$$

which completes the proof.

With the knowledge that $T(n)$ and $U(n)$ are expressible in terms of the $A_{k}(\alpha, \beta)$, we can use Lemma 3 to establish several more relationships and identities which will be helpful later.

LEMMA 4.

(a)

$$
\sum_{i+j=n} T(i) T(j)=\frac{5 n+1}{2 n+1} T(n)
$$

(b)

$$
\sum_{2 i+j=n} T(i) U(j)= \begin{cases}\frac{5 n+2}{2 n+2} U(n) & \text { (n even) } \\ \frac{5 n+1}{3 n+3} U(n) & (n \text { odd })\end{cases}
$$

(c)

$$
\sum_{i+j=n} U(i) U(j)= \begin{cases}A_{\frac{1}{2} n}(2,5)+A_{\frac{1}{2} n-1}(6,5) & (n \text { even }), \\ 2 A_{\frac{1}{2}(n-1)}(4,5) & (n \text { odd }) ;\end{cases}
$$

(d) $\sum_{i+j+k=n} T(i) T(j) T(k)=U(2 n+1)$.

(b) Enumeration formulas. We now derive for each of the automorphism groups a formula for the number of hexagon trees with that symmetry group. For a group $X$ in Table 2 we denote by $X(n)$ the number with $n$ hexagons. For convenience we use the term $X$-symmetric to describe trees with symmetry group $X$. For ease of computation we consider the groups in reverse order. 
$M$. Any $M$-symmetric hexagon tree is obtained if two isomorphic trees with reflectional symmetry ( $U$-type) are joined along their root edges. Thus

$$
M(n)=U\left(\frac{1}{2} n\right) \text {. }
$$

$L$. A hexagon tree with this type of symmetry is obtained by joining appropriately two isomorphic trees along their root edges. This of course yields also the $M$-symmetric trees. Further, each $L$-symmetric.tree is obtained in two ways since a given rooted tree and its mirror image yield the same $L$-symmetric tree. The desired formula is therefore

$$
L(n)=\frac{1}{2}\left[T\left(\frac{1}{2} n\right)-M(n)\right] .
$$

$K$. This case is similar to the previous one except that we join mirror images instead of isomorphic trees. The formula is the same.

$$
K(n)=\frac{1}{2}\left[T\left(\frac{1}{2} n\right)-M(n)\right] .
$$

$J$. This type of symmetry arises when isomorphic $U$-type hexagon trees are joined to the edges of a given hexagon. Hence

$$
J(n)=U\left(\frac{n-1}{6}\right) .
$$

I. In this case, the same rooted tree is joined to each of three alternate edges of a hexagon, while the mirror image of that tree is joined to each of the three remaining edges. This also yields those trees with $J$-symmetry, and each $I$-symmetric tree arises twice, so that

$$
I(n)=\frac{1}{2}\left[T\left(\frac{n-1}{6}\right)-J(n)\right] .
$$

$H$. To count these trees, we take two $U$-type trees and join each to three alternate edges of a given hexagon. This again counts the $J$-symmetric trees once and counts the $H$-symmetric trees twice since the two choices of rooted trees may be interchanged. The result is

$$
H(n)=\frac{1}{2}\left[\sum_{3(i+j)=n-1} U(i) U(j)-J(n)\right]
$$

By Lemma 4(c), we have

$$
H(n)= \begin{cases}A_{\frac{1}{b(n-4)}}(4,5)-\frac{1}{2} J(n) & \text { for } n \equiv 4(\bmod 6), \\ \frac{1}{2}\left[A_{\frac{1}{b(n-1)}}(2,5)+A_{\frac{t}{(n-7)}}(6,5)-J(n)\right] & \text { for } n \equiv 1(\bmod 6), \\ 0 & \text { otherwise. }\end{cases}
$$

$G$. If the same rooted tree is joined to each edge of a hexagon, a tree with $G$-symmetry is obtained. This is like the $I$-symmetric case in that the procedure also yields the $J$-symmetric trees and each desired tree twice, so that

$$
G(n)=\frac{1}{2}\left[T\left(\frac{n-1}{6}\right)-J(n)\right]
$$


$F$. To obtain the trees having this symmetry, we join a $U$-type tree to two opposite edges, an arbitrary tree to another pair of opposite edges, and the mirror image of the latter tree to the remaining pair. Since this also yields the $J$-symmetric trees, we have

$$
F(n)=\sum_{4 i+2 j=n-1} T(i) U(j)-J(n) .
$$

An application of Lemma 4(b) shows that

$$
F(n)= \begin{cases}\frac{5 n-1}{2 n+2} U\left(\frac{n-1}{2}\right)-J(n) & \text { for } \quad n \equiv 1(\bmod 4) \\ \frac{5 n-3}{3 n+3} U\left(\frac{n-1}{2}\right)-J(n) & \text { for } \quad n \equiv 3(\bmod 4) \\ 0 & \text { otherwise. }\end{cases}
$$

$E$. The $E$-symmetric trees are obtained by adding one rooted tree to three alternate edges and another to the remaining edges. Each of the two possible orientations of a tree with $G$-symmetry is obtained in this manner, while each $H$-symmetric and $I$-symmetric tree is obtained twice by the possible choices of the rooted trees. The $J$-symmetric trees also arise in this fashion, and the desired trees with $E$-symmetry are obtained four times (twice due to orientation and twice from choice of the rooted trees), which provides the result that

$$
E(n)=\frac{1}{4}\left[\sum_{3(i+j)=n-1} T(i) T(j)-2 G(n)-2 H(n)-2 I(n)-J(n)\right] .
$$

As a consequence of Lemma $4(a)$,

$$
E(n)=\frac{1}{4}\left[\frac{5 n-2}{2 n+1} T\left(\frac{n-1}{3}\right)-2 G(n)-2 H(n)-2 I(n)-J(n)\right] .
$$

D. A hexagon tree with $180^{\circ}$-rotational symmetry is obtained by selecting three rooted trees and joining each to two opposite edges of a hexagon. If this is done in all possible ways, those trees with $F$-symmetry are obtained three times (any of the three rooted trees may have the required reflectional symmetry), those with $G$-symmetry twice (due to orientation), and those with $J$-symmetry once. Further, each of the desired trees arises in six ways; so we have

by Lemma 4(d).

$$
\begin{aligned}
D(n) & =\frac{1}{6}\left[\sum_{2(i+j+k)=n-1} T(i) T(j) T(k)-3 F(n)-2 G(n)-J(n)\right] \\
& = \begin{cases}\frac{1}{6}[U(n)-3 F(n)-2 G(n)-J(n)] & (n \text { odd }), \\
0 & (n \text { even }),\end{cases}
\end{aligned}
$$

C. For a $C$-symmetric tree, one again takes three rooted hexagon trees but places them and their mirror images so as to obtain reflectional symmetry in a diagonal. This includes the $F_{-}, I$ - and $J$-symmetric trees, with the $I$-symmetric ones occurring twice, the others just once. The remaining trees occur twice. It follows that 


$$
\begin{aligned}
C(n) & =\frac{1}{2}\left[\sum_{2(i+j+k)=n-1} T(i) T(j) T(k)-F(n)-2 I(n)-J(n)\right] \\
& = \begin{cases}\frac{1}{2}[U(n)-F(n)-2 I(n)-J(n)] & (n \text { odd }), \\
0 & (n \text { even) } .\end{cases}
\end{aligned}
$$

$B$. This case is considered in a different way from the others. Each $B$-symmetric tree corresponds to two rooted hexagon trees with reflectional symmetry. However, the trees with $F$-, $H$-, $J$ - and $M$-symmetries also correspond to $U$-type trees, with the $H$-symmetric ones corresponding to two, the others to just one each (because of their " top-to-bottom" symmetries). Hence

$$
B(n)=\frac{1}{2}[U(n)-F(n)-2 H(n)-J(n)-M(n)] .
$$

$A$. Those hexagon trees having no nontrivial permutations are counted using all the others. The number of ways in which a hexagon tree with $n$ hexagons having a given symmetry can be rooted is twice $4 n+2$ divided by the order of its automorphism group. This is because there are in general two ways to put a tree in the plane and $4 n+2$ ways to choose the root edge; one divides by the order of the group to eliminate redundancies. Therefore the total number of rooted trees is

$$
T(n)=4(2 n+1) \sum_{X} \frac{X(n)}{\sigma(X)},
$$

where the sum is over all 13 automorphism groups, $\sigma(X)$ is the order of group $X$, and $X(n)$ is the number of hexagon trees with that group. As we know all quantities except $A(n)$, we obtain

$$
A(n)=\frac{T(n)}{4(2 n+1)}-\sum_{X \neq A} \frac{X(n)}{(\sigma) X}
$$

The following theorem provides a summary of these results.

THEOREM 2. The number of hexagon trees with $n$ hexagons and given automorphism group is as follows (where $A_{k}(\alpha, 5)=\frac{\alpha}{5 k+\alpha}\left(\begin{array}{c}5 k+\alpha \\ k\end{array}\right), T(k)=U(2 k)=A_{k}(1,5)$ and $U(2 k+1)=A_{k}(3,5)$ for $k$ an integer):

$$
\begin{aligned}
M(n) & =U\left(\frac{1}{2} n\right) . \\
L(n) & =\frac{1}{2}\left[T\left(\frac{1}{2} n\right)-M(n)\right] . \\
K(n) & =\frac{1}{2}\left[T\left(\frac{1}{2} n\right)-M(n)\right] . \\
J(n) & =U\left(\frac{n-1}{6}\right) . \\
I(n) & =\frac{1}{2}\left[T\left(\frac{n-1}{6}\right)-J(n)\right] .
\end{aligned}
$$

D 


$$
\begin{aligned}
& H(n)= \begin{cases}\frac{1}{2}\left[A_{\frac{1}{t}(n-1)}(2,5)+A_{\frac{1}{\delta}(n-7)}(6,5)-J(n)\right] & \text { for } n \equiv 1(\bmod 6), \\
A_{\frac{1}{t}(n-4)}(4,5)-\frac{1}{2} J(n) & \text { for } n \equiv 4(\bmod 6), \\
0 & \text { otherwise. }\end{cases} \\
& G(n)=\frac{1}{2}\left[T\left(\frac{n-1}{6}\right)-J(n)\right] \text {. } \\
& F(n)= \begin{cases}\frac{5 n-1}{2 n+2} U\left(\frac{n-1}{2}\right)-J(n) & \text { for } n \equiv 1(\bmod 4), \\
\frac{5 n-1}{3 n+3} U\left(\frac{n-1}{2}\right)-J(n) & \text { for } n \equiv 3(\bmod 4), \\
0 & \text { otherwise. }\end{cases} \\
& E(n)=\frac{1}{4}\left[\frac{5 n-2}{2 n+1} T\left(\frac{n-1}{3}\right)-2 G(n)-2 H(n)-2 I(n)-J(n)\right] \text {. } \\
& D(n)= \begin{cases}\frac{1}{6}[U(n)-3 F(n)-2 G(n)-J(n)] & \text { for } n \text { odd, } \\
0 & \text { for } n \text { even } .\end{cases} \\
& C(n)= \begin{cases}\frac{1}{2}[U(n)-F(n)-2 I(n)-J(n)] & \text { for } n \text { odd, } \\
0 & \text { for } n \text { even. }\end{cases} \\
& B(n)=\frac{1}{2}[U(n)-F(n)-2 H(n)-J(n)-M(n)] . \\
& A(n)=\frac{T(n)}{4(2 n+1)}-\sum_{X=B}^{M} \frac{X(n)}{\sigma(X)} .
\end{aligned}
$$

We now derive our main result.

THEOREM 3. The number $S(n)$ of planar hexagon trees is

$$
S(n)=\frac{1}{4(2 n+1)} T(n)+\frac{1}{6} T\left(\frac{1}{2} n\right)+\frac{5 n-2}{6(2 n+1)} T\left(\frac{n-1}{3}\right)+\frac{1}{6} T\left(\frac{n-1}{6}\right)+\frac{7}{12} U(n),
$$

where $T(x)$ is 0 if $x$ is not an integer, and otherwise

$$
\begin{array}{r}
T(n)=U(2 n)=\frac{1}{5 n+1}\left(\begin{array}{c}
5 n+1 \\
n
\end{array}\right), \\
U(2 n+1)=\frac{3}{5 n+3}\left(\begin{array}{c}
5 n+3 \\
n
\end{array}\right) .
\end{array}
$$

Proof. Since

$$
S(n)=\sum_{X=A}^{M} X(n)
$$


use of the above formula for $A(n)$ gives

$$
S(n)=\frac{T(n)}{4(2 n+1)}+\sum_{X=B}^{M} \frac{\sigma(X)-1}{\sigma(X)} X(n) .
$$

Substitution of the formulas for $B(n)$ to $M(n)$ yields

$$
S(n)=\frac{T(n)}{4(2 n+1)}+\frac{1}{2} T\left(\frac{1}{2} n\right)+\frac{5 n-2}{6(2 n+1)} T\left(\frac{n-1}{3}\right)+\frac{1}{6} T\left(\frac{n-1}{6}\right)+ \begin{cases}\frac{7}{12} U(n) & (n \text { odd }) \\ \frac{1}{4} U(n) & (n \text { even }) .\end{cases}
$$

However, use of the fact that $U(2 m)=T(m)$ and the fact that $T\left(\frac{1}{2} n\right)=0$ for $n$ odd, lets us replace $\frac{1}{3} T\left(\frac{1}{2} n\right)$ by $\frac{1}{3} U(n)$ in the even case, which simplifies the result.

4. Hexagon chains. Having enumerated a more general class of structures than polyhexes, we now turn to a more restricted class, the hexagon chains. We recall that these are the polyhexes in which each hexagon meets at most two others.

Because of the way hexagon chains are constructed, we utilize a much more restricted form of rooting than for hexagon trees; in this restricted form, the distinguished edge must be such that it is on an end hexagon and its vertices lie on only that hexagon. (A similar sort of restricted rooting is used for polyhexes.) It is readily seen that a nontrivial hexagon chain can have just two end hexagons, each with three possible root edges. Hence the number of possible rootings is 12 divided by the order of the automorphism group.

Let $t(n)$ denote the number of rooted hexagon chains, and $u(n)$ the number with symmetry in the root edge. It follows at once by induction that $t(n)=3^{n-1}$, since there are just three ways to adjoin a new hexagon to a given rooted hexagon chain. Clearly there is just one chain with $n$ hexagons and root symmetry. This establishes the following result.

THEOREM 4. The number of rooted hexagon chains with $n$ hexagons is

$$
t(n)=3^{n-1}
$$

The number with root symmetry is

$$
u(n)=1 .
$$

The possible automorphism groups for these structures are also more restricted than they are for hexagon trees. Observing that a single hexagon is a chain with automorphism group $J$, we note that this is the only chain with that group. Hence we confine our analysis to chains with two or more hexagons. We also note that each of the groups $E, G, H$ and $I$ is never the group of a chain. Therefore we have only eight groups (including the identity) to consider. To denote the number of hexagon chains with a given automorphism group, we use the corresponding lower-case letter. The following results are easily verified.

THEOREM 5. The number of hexagon chains with $n$ hexagons $(n \geqq 2)$ and given automorphism group is 


$$
\begin{aligned}
& m(n)=u\left(\frac{1}{2} n\right), \\
& k(n)=l(n)=\frac{1}{2}\left[t\left(\frac{1}{2} n\right)-u\left(\frac{1}{2} n\right)\right], \\
& f(n)=u\left(\frac{n-1}{2}\right), \\
& c(n)=d(n)=\frac{1}{2}\left[t\left(\frac{n-1}{2}\right)-u\left(\frac{n-1}{2}\right)\right], \\
& b(n)=t\left(\frac{n-1}{2}\right), \\
& a(n)=\frac{1}{12} t(n)-\frac{1}{2} t\left(\frac{1}{2} n\right)-t\left(\frac{n-1}{2}\right)+\frac{1}{4},
\end{aligned}
$$

where $t(n)=3^{n-1}$ and $u(n)=1$ if $n$ is an integer, and each is zero if $n$ is not an integer.

By adding the terms in Theorem 5, we obtain the following result.

THEOREM 6. The number $s(n)$ of hexagon chains with $n$ hexagons $(n \geqq 2)$ is

$$
s(n)= \begin{cases}\frac{1}{4}\left(3^{r-1}+1\right)^{2} & \text { if } n=2 r, \\ \frac{1}{4}\left(3^{r-1}+1\right)\left(3^{r}+1\right) & \text { if } n=2 r+1 .\end{cases}
$$

5. Polyhexes. We have considered both a generalization and a restriction of polyhexes. We now return to the polyhexes themselves, comparing their enumeration with that of the more general hexagon trees. Recall that polyhexes are those planar hexagon trees in which no vertex is on more than two hexagons. The enumeration of this subclass is more difficult and consequently there is a solution only in terms of a generating function. The solutions to the two problems have a number of similarities, one being that both begin with enumerating rooted structures. However there is a difference in difficulty; for hexagon trees, the precise number of rooted structures $(T(n)$ above) has been found, but this is not the case for rooted polyhexes.

Changing the notation of [6] slightly, we let $V(n)$ denote the number of polyhexes with $n$ hexagons which are rooted at an exterior edge whose vertices are on only one hexagon. Letting $S(n)$ and $D(n)$ denote the number of these in which the root hexagon is joined to just one or to two other hexagons, Harary and Read observe that, for $n \geqq 2, V(n)=S(n)+D(n)$, $S(n+1)=3 V(n)$ and $D(n+1)=\sum V(r) V(s)(r+s=n ; r, s \geqq 1)$. They then obtain their generating function for $v(x)=\sum V(n) x^{n}$ as

$$
v(x)=\frac{1}{2 x}[1-3 x-\sqrt{ }\{(1-x)(1-5 x)\}] .
$$

It would appear that a simple recursion formula for $V(n)$ was overlooked:

$$
V(n)=3 V(n-1)+\sum_{i=1}^{n-2} V(i) V(n-i-1)
$$


This does not of course fit into a generating function solution any better, but it might be more convenient for computation purposes.

The only subsequent difficulty encountered in applying the procedure of $\$ 3$ to polyhexes is due to the problem of rooting an arbitrary polyhex. A hexagon tree with $n$ hexagons, as observed above, can be rooted at any of the $4 n+2$ exterior edges; the number of possible root edges of a polyhex can vary from 6 to $\left[\frac{3}{2}(n+2)\right]$ (greatest integer), depending on how the hexagons are joined. Attempts to solve the problem using the various numbers of possible root edges have led to equations of such complexity that no solution seems to be forthcoming.

Other than this difficulty in finding the number of structures with the identity automorphism group, the enumeration by groups is easier for polyhexes than for hexagon trees, since fewer groups can occur. The next two theorems summarize the results for rooted and unrooted polyhexes.

THEOREM 7. The number $V(n)$ of rooted polyhexes with $n$ hexagons satisfies the following recurrence relation:

$$
V(n)=3 V(n-1)+\sum_{i=1}^{n-2} V(i) V(n-i-1)
$$

The number $W(n)$ with reflectional symmetry satisfies

$$
W(n)= \begin{cases}W(n-1)+V\left(\frac{n-1}{2}\right) & \text { for } n \text { odd, } \\ W(n-1) & \text { for } n \text { even. }\end{cases}
$$

Proof. The first was observed above; the second is immediate when one considers the two ways of joining polyhexes to a given rooted hexagon and getting reflectional symmetry.

The proof of the next result is analogous to that of our main result, and further details will be omitted. The groups are the same as those in $\$ 2$, and Figure 4 might again be very helpful. Here we denote the number with group $X$ by $X^{\prime}(n)$. As before, some of the formulas are given in terms of others.

THEOREM 8. The number of polyhexes with $n$ hexagons and given nonidentity automorphism groups is as follows.

For $n=1, J^{\prime}(n)=1$; all others are 0.

For $n \geqq 2$,

$$
\begin{aligned}
M^{\prime}(n) & =W\left(\frac{1}{2} n\right), \\
K^{\prime}(n) & =L^{\prime}(n)=\frac{1}{2}\left[V\left(\frac{1}{2} n\right)-M^{\prime}(n)\right], \\
G^{\prime}(n) & =I^{\prime}(n)=J^{\prime}(n)=0, \\
H^{\prime}(n) & =W\left(\frac{n-1}{3}\right), \\
F^{\prime}(n) & =W\left(\frac{n-1}{2}\right),
\end{aligned}
$$




$$
\begin{aligned}
E^{\prime}(n) & =\frac{1}{2}\left[V\left(\frac{n-1}{3}\right)-H^{\prime}(n)\right] \\
C^{\prime}(n) & =D^{\prime}(n)=\frac{1}{2}\left[V\left(\frac{n-1}{2}\right)-F^{\prime}(n)\right], \\
B^{\prime}(n) & =\frac{1}{2}\left[\sum_{2 r+s=n} V(r) W(s)-F^{\prime}(n)-2 H^{\prime}(n)-M^{\prime}(n)\right] \\
& =\frac{1}{2}\left[V\left(\frac{1}{2} n\right)+3 V\left(\frac{n-1}{2}\right)-F^{\prime}(n)-2 H^{\prime}(n)-M^{\prime}(n)\right] .
\end{aligned}
$$

Rather than consider separately certain of the groups, one can combine the results according to the type of group and obtain the following enumeration result.

THEOREM 9. The number of unrooted polyhexes with $n$ hexagons $(n>1)$ and having each of the possible types of nontrivial automorphism groups is the following.

The symmetries of a triangle:

$$
\begin{aligned}
& W\left(\frac{n-1}{3}\right) . \\
& \frac{1}{2}\left\{V\left(\frac{n-1}{3}\right)-W\left(\frac{n-1}{3}\right)\right\} .
\end{aligned}
$$

Rotations of $120^{\circ}$ and $240^{\circ}$ :

A pair of reflections:

$$
W\left(\left[\frac{1}{2} n\right]\right) \text {. }
$$

A rotation of $180^{\circ}$ :

$$
\frac{1}{2}\left\{V\left(\left[\frac{1}{2} n\right]\right)-W\left(\left[\frac{1}{2} n\right]\right)\right\} \text {. }
$$

A single reflection:

$$
V\left(\frac{1}{2} n\right)+2 V\left(\frac{n-1}{2}\right)-W\left(\left[\frac{1}{2} n\right]\right)-W\left(\frac{n-1}{3}\right) \text {. }
$$

Here $V(x)$ and $W(x)$ are 0 if $x$ is not an integer; otherwise they are given recursively in Theorem $7 ;[x]$ denotes as usual the greatest integer function.

We conclude with a table comparing the numbers of hexagon chains, polyhexes, and hexagon trees for small values of $n$.

TABLE 3. Numbers of Structures WTth UP to 12 HeXagons

$\begin{array}{crrr}\begin{array}{c}\text { Number of } \\ \text { hexagons }\end{array} & \text { Hexagon chains } & \text { Polyhexes } & \text { Hexagon trees } \\ 1 & 1 & 1 & 1 \\ 2 & 1 & 1 & 1 \\ 3 & 2 & 2 & 3 \\ 4 & 4 & 5 & 12 \\ 5 & 10 & 12 & 68 \\ 6 & 25 & 37 & 483 \\ 7 & 70 & 123 & 3,946 \\ 8 & 196 & 446 & 34,485 \\ 9 & 574 & 1,689 & 315,810 \\ 10 & 1,681 & 6,693 & 2,984,570 \\ 11 & 5,002 & 27,034 & 28,907,970 \\ 12 & 14,884 & 111,630 & 285,601,251\end{array}$




\section{REFERENCES}

1. L. W. Beineke and R. E. Pippert, The number of labeled dissections of a $k$-ball, Math. Ann. 191 (1971), 87-98.

2. L. W. Beineke and R. E. Pippert, A census of ball and disk dissections, Graph Theory and Applications (Y. Alavi, D. R. Lick and A. T. White, editors) (Springer-Verlag, New York, 1972), $25-40$.

3. L. W. Beineke and R. E. Pippert, Enumerating dissectible polyhedra by their automorphism groups, Canad. J. Math. 26 (1974), 50-67.

4. R. K. Guy, Dissecting a polygon into triangles, Bull. Malayan Math. Soc. 5 (1958), 57-60. Research Paper No. 9, The University of Calgary, 1967. 122.

5. F. Harary and E. M. Palmer, On acyclic simplicial complexes, Mathematika 15 (1968), 115-

6. F. Harary and R. C. Read, The enumeration of tree-like polyhexes, Proc. Edinburgh Math. Soc. 17 (1970), 1-13.

7. T. Motzkin, Relations between hypersurface cross ratios and a combinatorial formula for partitions of a polygon, for permanent preponderance, and for non-associative products, Bull. Amer. Math. Soc. 54 (1948), 352-360.

8. E. M. Palmer, Variations of the cell growth problem, Graph Theory and Applications (Y. Alavi, D. R. Lick and A. T. White, editors) (Springer-Verlag, New York, 1972), $215-224$.

9. J. Riordan, Combinatorial identities (New York, 1968).

Purdub University at Fort WAYNe

FORT WAYNE, INDIANA, U.S.A. 